ARTÍCULO ORIGINAL

\title{
Barreras arquitectónicas en establecimientos sanitarios contribuyen a la discapacidad en Honduras
}

\author{
Architectural barriers in healthcare buildings contribute to disabilities in Honduras
}

\author{
Enma Molina Amaya, ${ }^{1}$ Ana Jiménez-Dilworth, ${ }^{2}$ Wilmer Castellanos-Dubón, ${ }^{3}$ Sonia Flores, ${ }^{4}$ Edna Maradiaga, ${ }^{5}$ Mauricio \\ Gonzáles $^{6}$, Jackeline Alger. ${ }^{7}$
}

${ }^{1}$ Médico Especialista en Medicina de Rehabilitación, Maestría en Métodos Cuantitativos de investigación en epidemiología; Departamento de Rehabilitación, Facultad de Ciencias Médicas (FCM), Universidad Nacional Autónoma de Honduras (UNAH); Tegucigalpa.

${ }^{2}$ Médico Residente, UNAH 2012-2014. Actualmente Especialista en Medicina de Rehabilitación; Fundación Teletón; Tegucigalpa.

${ }_{3}^{3}$ Médico Residente UNAH 2012-2014. Actualmente Especialista en Medicina de Rehabilitación, Departamento de Rehabilitación FCM UNAH; Fundación Teletón; San

Pedro Sula.

${ }^{4}$ Médico Especialista en Medicina de Rehabilitación; Departamento de Rehabilitación, Instituto Hondureño de Seguridad Social; Tegucigalpa.

${ }^{5}$ Médico Especialista Salud Pública; Unidad de Investigación Científica (UIC), FCM UNAH; Tegucigalpa.

'Licenciado en informática; Unidad de Investigación Científica (UIC), FCM UNAH; Tegucigalpa.

${ }^{7}$ Médico, Doctorado de Filosofía (PhD) en Parasitología; UIC FCM UNAH; Tegucigalpa.

RESUMEN. Antecedentes. Cobertura Universal en Salud es uno de los Objetivos de Desarrollo Sostenible. Las personas con discapacidad suelen tener mayores dificultades de acceso a la salud que el resto de la población. Factores del entorno como el diseño y las construcciones pueden ser una barrera o un facilitador. Objetivo. Evaluar las características de accesibilidad en el diseño y las ayudas técnicas para la movilidad en los establecimientos de salud en zonas de realización del servicio médico social, Carrera de Medicina UNAH, cohorte 2013-2014. Métodos. En base a principios de diseño universal se evaluaron barreras y facilitadores para el acceso a la salud en establecimientos de salud, incluyendo centros de atención primaria y hospitales. Resultados. Se evaluaron 176 establecimientos en 17 departamentos, 154 unidades de atención primaria (UAP) y 22 hospitales. Las mayores barreras fueron la señalización inadecuada de rutas: $150(97.4 \%)$ UAP y $20(90.9 \%)$ hospitales; la carencia de soportes en los baños: 152 (98.7\%) UAP y 20 (90.9\%) hospitales; la inaccesibilidad a personas en sillas de ruedas: $129(83.8 \%)$ UAP y $9(40.9 \%)$ hospitales. Discusión. Mejorar el acceso y la calidad de los servicios de salud para las personas con discapacidad es imprescindible para reducir las inequidades. Nuestro estudio indica que el diseño de los establecimientos de salud en Honduras supone una barrera para el acceso y calidad de salud de las personas con discapacidad. Se exhorta al Estado a regular y supervisar el diseño y construcción de edificios accesibles para todos, a fin de reducir las brechas en salud.

Palabras clave: Accesibilidad arquitectónica, Accesibilidad a los servicios de salud, Cobertura Universal de Salud, Infecciones por coronavirus, Servicios de salud para personas con discapacidad.

Recibido: 17-09-2020 Aceptado para publicación 15-12-2020

Dirección para correspondencia: Dra. Enma Molina Amaya

Correo electrónico: enma.molina@unah.edu.hn

Declaración de relaciones y actividades inancieras y no inancieras y con lictos de interés: ninguno.

DOI: $10.5377 /$ rmh.v88i2.11486

\section{INTRODUCCIÓN}

Se estima que alrededor del mundo más de 15 millones de personas viven con discapacidad y la mayoría de ellas vive en países de medianos y bajos ingresos. ${ }^{1}$ En Honduras la prevalencia de discapacidad en las personas jóvenes ronda entre 2.6\%-4.6\%., ${ }^{2,3}$ Entre los Objetivos de Desarrollo Sostenible (ODS), se encuentra la Cobertura Universal en Salud. Esto significa que todas las personas y las comunidades tengan acceso equitativo a los servicios que necesitan, con calidad y sin dificultades financieras. ${ }^{4}$ Las personas con discapacidad son un grupo vulnerable, pues en general tienen mayores dificultades de acceso a la salud y gastan más para obtener estos servicios, además se conoce que suelen ser de menor calidad que la necesitada. ${ }^{1}$ En un estudio para caracterizar la situación de las personas con discapacidad en Francisco Morazán, ${ }^{5}$ se encontró que la mayoría de las personas con discapacidad no accedía a ningún servicio de salud para la atención de sus problemas sanitarios y entre los que sí accedían, la mayoría acudía a la atención pública.

Los Estados miembros de la Organización de Naciones Unidas en la Convención de los Derechos de las Personas con Discapacidad se han comprometido a eliminar las barreras de acceso a los diferentes entornos, incluidos los establecimientos de salud, así como asegurar el acceso a los servicios de salud de calidad adecuada a sus necesidades. ${ }^{6}$ Según la Clasificación Internacional del Funcionamiento y la Discapacidad (CIF), ${ }^{7}$ facilitadores son aspectos personales o del ambiente que cuando están presentes mejoran el funcionamiento y reducen la discapacidad, mientras que barreras son aquellas que limitan el funcionamiento y generan discapacidad. De esta forma el diseño y equipamiento de los edificios públicos, incluidos aquellos que prestan servicios de salud son un ejemplo de estos factores ambientales, que de acuerdo a su presencia o ausencia pueden facilitar o limitar el desempeño y por lo tanto el acceso a la salud 
de las personas con discapacidad; se encuentran codificados de forma detallada en el capítulo 1 de los Factores Ambientales de la CIF: Productos y Tecnología. ${ }^{7}$ Por su parte, el diseño universal es un concepto que va más allá de la eliminación de barreras: trata de que los servicios, equipos y entornos, puedan ser usados por todos, de forma autónoma y segura, basándose en siete principios: puede ser usado por todos, se adapta a diferentes capacidades, el diseño es simple e intuitivo, la información es perceptible de manera eficaz para todas las personas, tolerancia al error / minimiza los riesgos, bajo esfuerzo físico para su uso y el espacio es suficiente para el uso y alcance de todas las personas. ${ }^{8}$

El objetivo del presente estudio fue realizar una evaluación cuantitativa de las características de diseño relacionadas con accesibilidad y las ayudas técnicas para la movilidad en las instalaciones de los Establecimientos de Salud donde había al menos un estudiante del último año de la Carrera de Medicina de la Universidad Nacional Autónoma de Honduras (UNAH) que participaban del estudio "Prevalencia de Discapacidad y sus Características en Población de 18 a 65 años, Honduras, Centro América, 2013-2014". ' 'La evaluación fue centrada en los siguientes principios del Diseño Universal: utilidad para todos, se adapta a las diferentes capacidades, información eficaz para todos y minimiza los riesgos. Los resultados de este estudio permitirán valorar las necesidades en el diseño y construcción de los edificios destinados a la prestación de servicios sanitarios a fin de que puedan ser usados por todos y disminuir las inequidades en el acceso y contribuir a mejorar la calidad de la salud de las personas con discapacidad en Honduras.

\section{METODOLOGÍA}

Se diseñó un estudio observacional de corte transversal. En base a principios de diseño universal se evaluaron las características relacionadas al diseño y productos de asistencia en Establecimientos de Salud donde estuvo asignado algún estudiante de último año de la Carrera de Medicina de la Facultad de Ciencias Médicas (FCM), UNAH, durante enero 2013- enero 2014. Esta evaluación fue parte de un estudio más amplio para determinar la prevalencia y posibles factores asociados a la discapacidad en sujetos de 18 - 65 años de edad. ${ }^{3}$ Los establecimientos estaban distribuidos en 17 departamentos del todo el país e incluyó todos los niveles asistenciales de la Secretaría de Salud (SESAL). De acuerdo a su nivel de complejidad la SESAL categoriza a las unidades de salud de atención primaria (UAP) como: Centro de Salud Rural (CSR), Centro Médico Odontológico (CMO) y Clínica Materno Infantil (CMI), y las categorías del nivel hospitalario: Hospital de Área (HA), Hospital Regional (HR) y Hospital Nacional (HN).

La evaluación fue centrada en los siguientes principios del Diseño Universal: utilidad para todos, se adapta a las diferentes capacidades, información eficaz para todos y minimiza los riesgos. Mediante observación directa y encuesta tipo entrevista al personal de salud responsable de los establecimientos, se recolectó la información que fue registrada en una lista de cotejo diseñada para tal fin. Las variables fueron las características observadas y se categorizaron de forma dicotómica: facilitador si estaba presente, o barrera en caso de no estar. Para el análisis y resultados, las características observadas se clasificaron de acuerdo al principio de diseño universal al que mejor hace referencia: ${ }^{9}$ A. Utilidad para todos: se observó la presencia de rampas de acceso y en el interior, aceras, pasillos de ancho suficiente para libre circulación incluso en silla de ruedas (más de $180 \mathrm{~cm}$ ) y puertas internas y exteriores con ancho suficiente para que pase una silla de ruedas $(90 \mathrm{~cm})$, B. Se adapta a diferentes capacidades: existencia de ayudas técnicas para la movilidad como bastones, andadores, sillas de ruedas, etc., C. La información es eficaz para todos: la señalización de rutas y lugares con letreros grandes y luminosos o con contraste de colores que permita su lectura a todas las personas y D. Minimiza los riesgos: existen barras de soporte en los baños y pasamanos en los pasillos.

Adicionalmente, para identificar la accesibilidad a los usuarios en sillas de ruedas se creó una variable que agrupó la presencia de rampas y de puertas y pasillos con ancho adecuado para el paso de sillas de ruedas. En caso de cumplir las tres características se consideró un facilitador y en caso de no cumplir una o más características se consideró una barrera. La lista de chequeo incluyó dos preguntas relacionadas con la preparación del personal de salud y programas orientados a la prevención y atención de las personas con discapacidad, estas no se analizaron ya que este estudio se centró en el diseño y las ayudas técnicas para la movilidad en los establecimientos de salud.

El análisis se realizó en el programa Stata versión 14. Se realizaron tablas de doble entrada para estimar frecuencias y porcentajes de las variables estudiadas. El estudio contó con dictamen del Comité de Ética en Investigación Biomédica (CEIB) FCM-UNAH. Los investigadores, estudiantes del último año de la Carrera de Medicina, residentes del Postgrado de Medicina Física y Rehabilitación y docentes de la Facultad de Ciencias Médicas y especialistas en medicina física y rehabilitación, completaron el curso en línea Conducta Responsable en la Investigación y/o Curso de Buenas Prácticas Clínicas del Programa CITI, Universidad de Miami (https://www.citiprogram. org).

\section{RESULTADOS}

Se evaluó un total de 189 establecimientos, 13 (6.9\%) de ellas se excluyeron del análisis por información incompleta. Se analizaron 176 listas de cotejo correspondientes a Establecimientos de todos los niveles asistenciales, distribuidos en 17 de 18 Departamentos del país (Figura 1). Se evaluaron 154 UAP, que representan el $9.6 \%$ de las UAP registradas en la Secretaría de Salud y 22 hospitales, el $71 \%$ del total nacional (Cuadro 1). Ninguno de los establecimientos contaba con la totalidad de las características evaluadas.

El Cuadro 2 detalla los aspectos de Diseño Universal encontrados como Barreras o Facilitadores en los Establecimientos de Salud. Entre las características de utilidad para todos, se encontró que la falta de rampas, se mostró como barrera en $119(77.3 \%)$ de las 154 UAP, mientras que el ancho de puertas 


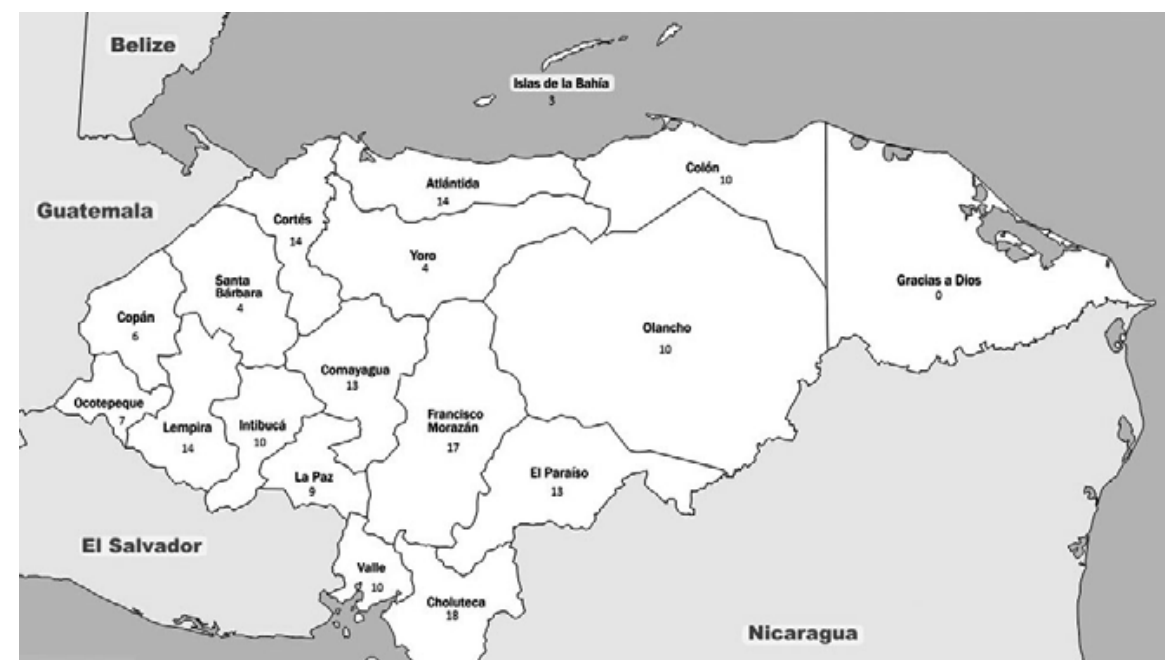

Figura 1. Número de establecimientos de salud evaluados por accesibilidad según Departamento, Honduras, 2014.

Cuadro 1. Proporción de establecimientos de salud evaluados por accesibilidad Honduras, 2014, $\mathrm{n}=176$.

\begin{tabular}{|c|c|c|c|}
\hline \multirow[t]{3}{*}{ Nivel asistencial } & \multicolumn{3}{|c|}{ Establecimientos de Salud } \\
\hline & \multirow{2}{*}{$\begin{array}{l}\text { A nivel Nacional* } \\
\mathrm{N}\end{array}$} & \multicolumn{2}{|c|}{ Evaluados } \\
\hline & & $\mathrm{N}$ & $(\%)$ \\
\hline \multicolumn{4}{|l|}{ Atención Primaria } \\
\hline CSR & 1089 & 35 & (3.2) \\
\hline $\mathrm{CMO}$ & 445 & 101 & $(22.7)$ \\
\hline CMI & 74 & 18 & $(24.3)$ \\
\hline Total & 1608 & 154 & $(9.6)$ \\
\hline \multicolumn{4}{|l|}{ Hospitales } \\
\hline Hospital de Área & 17 & 15 & $(88.2)$ \\
\hline Hospital Regional & 6 & 6 & $(100.0)$ \\
\hline Hospital Nacional & 8 & 1 & $(12.5)$ \\
\hline Total & 31 & 22 & $(71.0)$ \\
\hline
\end{tabular}

* De acuerdo al Registro de Unidades Prestadoras de Servicio de la Secretaría de Salud en 2017. fue un facilitador en 125 de estos establecimientos (81.2\%). Se encontraron barreras para adaptarse a diferentes capacidades: en $127(82.5 \%)$ no había ayudas técnicas para la movilidad. No había señalización adecuada en 150 (97.4\%) de las UAP, lo cual fue una barrera para la información eficaz para todos. Las barreras para minimizar los riesgos fueron: 152 (98.7\%) UAP sin soportes en los baños y 148 (96.1\%) UAP sin pasamanos en los pasillos.

El Cuadro 3 detalla las características del Diseño Universal en los Hospitales. La característica de utilidad para todos que se encontró como mayor facilitador fueron las aceras, que estaban presentes en 16 (86.4\%) de los 22 hospitales en el estudio; el ancho de los pasillos y el ancho de puertas -cada uno- fue barrera en $7(31.8 \%)$ hospitales. La presencia de ayudas técnicas para la movilidad se encontró como facilitador para adaptarse a diferentes capacidades en $16(72.7 \%)$ de estos estable-

Cuadro 2. Características de diseño universal identificadas como barreras o facilitadores para la accesibilidad en los establecimientos de salud de atención primaria, Honduras, 2014, n=154

\begin{tabular}{|c|c|c|c|c|}
\hline \multirow{2}{*}{ Característica } & \multicolumn{2}{|c|}{ Barrera } & \multicolumn{2}{|c|}{ Facilitador } \\
\hline & $\mathrm{N}$ & $(\%)$ & $\mathrm{N}$ & $(\%)$ \\
\hline \multicolumn{5}{|l|}{ Utilidad para todos } \\
\hline Rampas & 119 & $(77.3)$ & 35 & $(22.7)$ \\
\hline Aceras & 45 & $(29.2)$ & 109 & $(70.8)$ \\
\hline Pasillos anchos (más de $180 \mathrm{~cm}$ ) & 70 & $(45.4)$ & 85 & $(55.2)$ \\
\hline Puertas anchas (más de $90 \mathrm{~cm}$ ) & 29 & $(18.8)$ & 125 & $(81.2)$ \\
\hline \multicolumn{5}{|l|}{ Se adapta a diferentes capacidades } \\
\hline $\begin{array}{l}\text { Ayudas técnicas para la movilidad (bastones, } \\
\text { andadores, sillas de ruedas) }\end{array}$ & 127 & $(82.5)$ & 27 & $(17.5)$ \\
\hline \multicolumn{5}{|l|}{ Información eficaz para todos } \\
\hline Adecuada señalización de rutas y lugares & 150 & $(97.4)$ & 4 & $(2.6)$ \\
\hline \multicolumn{5}{|l|}{ Minimiza riesgos } \\
\hline Soporte en los baños & 152 & $(98.7)$ & 2 & $(1.3)$ \\
\hline Pasamanos en los pasillos & 148 & $(96.1)$ & 6 & $(3.9)$ \\
\hline
\end{tabular}


Cuadro 3. Características de diseño universal identificadas como barreras o facilitadores para la accesibilidad en los establecimientos de salud a nivel hospitalario, Honduras, 2014, n=22

\begin{tabular}{|c|c|c|c|c|}
\hline \multirow{2}{*}{ Característica } & \multicolumn{2}{|c|}{ Barrera } & \multicolumn{2}{|c|}{ Facilitador } \\
\hline & $\mathrm{N}$ & $(\%)$ & $\mathrm{N}$ & $(\%)$ \\
\hline \multicolumn{5}{|l|}{ Utilidad para todos } \\
\hline Rampas & 6 & $(27.3)$ & 16 & $(72.7)$ \\
\hline Aceras & 3 & $(13.6)$ & 19 & $(86.4)$ \\
\hline Pasillos anchos (más de $180 \mathrm{~cm}$ ) & 7 & $(31.8)$ & 15 & $(68.2)$ \\
\hline Puertas anchas (más de $90 \mathrm{~cm}$ ) & 7 & $(31.8)$ & 15 & $(68.2)$ \\
\hline \multicolumn{5}{|l|}{ Se adapta a diferentes capacidades } \\
\hline $\begin{array}{l}\text { Ayudas técnicas para la movilidad bastones, } \\
\text { andadores, sillas de ruedas) }\end{array}$ & 6 & $(27.3)$ & 16 & $(72.7)$ \\
\hline \multicolumn{5}{|l|}{ Información eficaz para todos } \\
\hline Adecuada señalización de rutas y lugares & 20 & $(90.9)$ & 2 & (9.1) \\
\hline \multicolumn{5}{|l|}{ Minimiza riesgos } \\
\hline Soporte en los baños & 20 & $(90.9)$ & 2 & (9.1) \\
\hline Pasamanos en los pasillos & 18 & $(81.8)$ & 4 & $(18.2)$ \\
\hline
\end{tabular}

Cuadro 4. Barreras para el acceso a usuarios con sillas de ruedas en los establecimientos de salud de Honduras, 2014, $n=176$

\begin{tabular}{|c|c|c|c|c|}
\hline \multirow{3}{*}{ Nivel asistencial de las unidades de salud } & \multicolumn{4}{|c|}{ Diseño accesible para usuarios en sillas de ruedas } \\
\hline & \multicolumn{2}{|c|}{ No } & \multicolumn{2}{|c|}{ Si } \\
\hline & $\mathrm{N}$ & $(\%)$ & $\mathrm{N}$ & $(\%)$ \\
\hline \multicolumn{5}{|l|}{ Atención primaria } \\
\hline CSR & 31 & $(88.6)$ & 4 & $(11.4)$ \\
\hline $\mathrm{CMO}$ & 86 & $(85.2)$ & 15 & $(14.8)$ \\
\hline $\mathrm{CMI}$ & 12 & $(66.7)$ & 6 & $(33.3)$ \\
\hline Total & 129 & $(83.8)$ & 25 & $(16.2)$ \\
\hline \multicolumn{5}{|l|}{ Hospitales } \\
\hline Hospital de Área & 8 & $(53.4)$ & 7 & $(46.6)$ \\
\hline Hospital Regional & 1 & $(16.7)$ & 5 & $(83.3)$ \\
\hline Hospital Nacional & 0 & $(0.0)$ & 1 & $(100.0)$ \\
\hline Total & 9 & $(40.9)$ & 13 & $(59.1)$ \\
\hline
\end{tabular}

cimientos. Las barreras más frecuentes fueron para la información eficaz y para minimizar riesgos: se encontraron 20 hospitales $(90.9 \%)$ sin adecuada señalización de rutas y en igual número faltaban soportes en los baños. El Cuadro 4 muestra las barreras para el acceso de personas en sillas de ruedas a los Establecimientos de los diferentes niveles asistenciales. Se encontró que 129 (83.8\%) de las 154 UAP y 9 (40.9\%) de los 22 hospitales no eran accesibles a personas en sillas de ruedas.

\section{DISCUSIÓN}

Este estudio valoró las características mínimas de diseño universal en 176 Establecimientos de la red pública asistencial en Honduras. El principal hallazgo fue que ninguno de los establecimientos contaba con la totalidad de las características evaluadas. Más del $90 \%$ de los establecimientos evaluados no están diseñados para minimizar los riesgos ni para que la información sea eficaz para todos, por lo cual son una barrera para el uso autónomo por personas con diferentes limitaciones físicas y sensoriales. La presencia conjunta de rampas, puertas y pasillos de ancho suficiente solo facilitaba el acceso para usuarios en sillas de rueda en 25 de 154 UAP y solo 27 de estos establecimientos contaban con ayudas técnicas para la movilidad, lo cual indica que son inaccesibles para personas con movilidad reducida. De igual manera, la mayoría de los hospitales no están diseñados para facilitar el acceso a personas con movilidad reducida pues solo 16 de 22 contaba con ayudas técnicas para la movilidad y 13 permitían la movilidad autónoma de usuarios de sillas de ruedas.

El diseño universal pretende que los entornos físicos y los sistemas puedan ser usados por la mayor parte de las personas, cualesquiera que sean sus capacidades. ${ }^{8}$ Este concepto se basa en siete principios que engloban las características esenciales que debe regir el diseño de cualquier espacio o sistema 
que sea de uso humano, de forma que puedan ser aprovechados por todas las personas de manera autónoma y segura. Por su parte, el término accesibilidad es un concepto que cualifica las características del entorno en relación a las características de un grupo de personas. Así, un lugar accesible es aquel que permite que un grupo de personas con ciertas características puedan encontrarse en él y un lugar no accesible es aquel que tiene barreras para que este grupo de personas puedan estar en él. ${ }^{9}$ Las acciones encaminadas a mejorar el acceso y la calidad de los servicios de salud que necesitan las personas con discapacidad son un factor imprescindible para reducir las inequidades y lograr las cobertura universal en salud. ${ }^{10} \mathrm{La}$ eliminación de barreras arquitectónicas está contemplada en la legislación de la mayoría de los países, pero su cumplimiento es incompleto o inadecuado.

En Honduras desde el año 2005 está vigente la Ley de Equidad y Desarrollo Integral para las Personas con Discapacidad. ${ }^{11}$ Esta ley establece como un derecho la igualdad de oportunidades para acceder a todos los servicios públicos. En el 2010, a través de la Dirección General de Desarrollo para las Personas con Discapacidad (DIGEDEPDI) se estableció un Plan Nacional de Accesibilidad Universal cuyo primer objetivo es "aclarar las obligaciones de las instituciones públicas, responsables para garantizar a las personas con discapacidad una adecuada accesibilidad, tanto a su entorno físico como a la tecnología de la información y la comunicación". ${ }^{12} \mathrm{~A}$ pesar de ello, los hallazgos de nuestro estudio indican que el diseño de los Establecimientos de la red pública en todos los niveles asistenciales supone una barrera para que las personas con discapacidad puedan acceder a la atención sanitaria que necesitan, de manera autónoma y segura. Hallazgos similares tuvieron Frost y cols. ${ }^{13}$ en una ciudad de Estados Unidos, donde las normativas de los requerimientos de accesibilidad son muy precisas. Ellos evaluaron el grado de cumplimiento de las normas de accesibilidad en 25 unidades especializadas de salud y 5 centros de atención primaria, encontrando que $83 \%$ de los baños de dichas instituciones no cumplía los requerimientos y $93 \%$ de los consultorios no cumplía con una o más de las características exigidas por la normativa de su país. En América Latina, Brasil es el país que puntea los estudios al respecto. Vinholes Siqueira y cols. ${ }^{14}$ estudiaron 240 unidades básicas de salud en siete Estados y encontraron barreras en el acceso por ausencia de rampas en $64 \%$ de los establecimientos y falta de aceras en $69 \%$ de los mismos. De Figueiredo Carvalho y cols. ${ }^{15}$ evaluaron las clínicas y consultorios de Neurología de la ciudad de Fortaleza e identificaron que cerca de la mitad de las clínicas carece de rampas, el $82 \%$ carece de pasamanos doble, casi el $20 \%$ de las clínicas y consultorios no cuenta con puertas de anchura suficiente para el paso de sillas de ruedas. En cambio, un estudio de cuatro hospitales públicos en un municipio de Paraíba ${ }^{16}$ a pesar de haber barreras importantes, en general mostró un mejor panorama: $75 \%$ tenía rampas de acceso y el 100\% tenía áreas comunes de circulación y puertas con un ancho adecuado; además se encontró que la mitad de los hospitales tenía barras de seguridad en los baños. En contraste con estos hallazgos, un estudio diagnóstico de la accesibilidad en Hospitales del País
Vasco, España se evidencia como la accesibilidad es un componente relevante y va más allá de identificar la existencia de rampas 0 ancho adecuado de puertas, sino que además incluye la accesibilidad urbanística de los alrededores, lo cual también es fundamental para el acceso a la salud. ${ }^{17}$

Alrededor del mundo 400 millones de personas no tienen acceso a los servicios de salud básicos. ${ }^{18}$ Un grupo particularmente vulnerable para estar en esta situación son las personas con discapacidad ya que las limitaciones físicas, mentales 0 sensoriales se suman y agudizan las dificultades acarreadas por la pobreza y los aspectos geográficos. Las principales barreras que encuentran las personas con discapacidad para el acceso a la atención primaria en las áreas rurales son causadas por la falta de disponibilidad de recursos humanos, de infraestructura y de suministro de equipo médico y auxiliar; la situación geográfica pues requiere movilizarse en silla de ruedas hasta el lugar de la atención; asequibilidad monetaria ya que aunque los servicios sean gratuitos, no tienen dinero para costear los medicamentos y los insumos para cuidados personales; adaptabilidad de los espacios ya que la mayoría de los establecimientos tiene numerosas barreras para el acceso, para movilizarse dentro de ellos y para el uso de los espacios y muebles de examinación; así mismo la falta de conocimiento de los proveedores de salud y de los que diseñan las políticas sanitarias acerca de las necesidades de las personas con discapacidad es un factor que limita el acceso y disminuye la calidad de los servicios de atención primaria. ${ }^{19}$

En Honduras, a pesar que el Modelo de Salud ${ }^{20}$ contempla entre sus principios la equidad y la calidad en los servicios, en 2010 solo el $37.2 \%$ de los asalariados contaban con cobertura de salud ${ }^{21}$ y como en el resto del mundo, las personas con discapacidad están en una posición muy desfavorecida: la cobertura de servicios es muy baja -en Francisco Morazán $77.3 \%$ de las personas con discapacidad no tienen acceso a ningún servicio de salud para atender sus problemas sanitarios $-{ }^{5}$ y nuestros datos reflejan calidad sub-óptima en los servicios, ya que para lograr la atención en la mayoría de los Establecimientos analizados, necesariamente deben sortear numerosas barreras para el acceso y el desplazamiento dentro de los edificios.

A diez años del plazo para el cumplimiento de los ODS falta mucho por recorrer en cuanto a equidad en salud, específicamente en lo referente a la salud de las personas con discapacidad y, la actual pandemia producida por el nuevo coronavirus SARS-CoV-2 (COVID-19) lo ha hecho relucir de diferentes maneras, ${ }^{22,23}$ tan solo pensando en las barreras físicas nos vemos obligados a preguntarnos; ¿Cómo se puede guardar distancia social si se necesita ser cargado para movilizarse en los diferentes niveles de un edificio donde no hay rampas? ¿Cómo lavarse las manos si los lavabos son alcanzables solo para quienes pueden estar de pie? Sin embargo, la actual crisis también puede ser una ocasión para repensar en cómo el espacio físico puede ser un componente que favorezca el control de la pandemia -y otras enfermedades- y al mismo tiempo pueden ser sostenibles ${ }^{24} \mathrm{y}$ accesibles para todos; por ejemplo, pasillos más amplios pueden permitir la distancia física entre las personas y mejoran la movilidad de las personas con limitaciones físicas, la 
iluminación natural además de reducir el gasto puede mejorar la salud mental de las personas y favorecer a las personas con visión reducida, las puertas automáticas evitan la propagación del virus y son accesibles para todos, etc. Así como el control de la pandemia de COVID-19 se concibe como un bien común y se entiende razonablemente que será solo posible con el compromiso de todos los sectores, también la reducción de inequidades en salud y la eliminación de barreras para las personas con discapacidad es un bien para todos y será posible solo por la implicación de todos.

Nuestro estudio tiene ciertas limitaciones, la primera es que solo se tomaron en cuenta las características de accesibilidad de los edificios y no la accesibilidad urbanística en donde se encuentran los mismos, además solo se apreció si los Establecimientos tenían o no las características en cuestión y no se dio un valor a las cualidades en relación a una norma o a la percepción de calidad por los usuarios ni se identificó si las dificultades son para el ingreso o para hacer uso de los edificios al interior. Sin embargo, hemos basado nuestra lista de cotejo en los principios del Diseño Universal, considerando las características mínimas razonables para el ingreso y la movilidad de todas las personas, incluidas aquellas con movilidad reducida y otras limitaciones funcionales; a su vez, dichas características están contempladas dentro del capítulo de Productos y Tecnologías de $\mathrm{CIF}^{7}$ lo cual permite contar con una mirada general de la situación y su comparación con los hallazgos de otros estudios. Otra limitación es que se ha hecho mayor énfasis en las características físicas y de equipamiento y menos a la comunicación y señalización, con lo cual solo se visualiza parcialmente las barreras o facilitadores para las personas con discapacidad sensorial. A pesar de ello, debido a que los parámetros para valorar esto fueron los menos exigentes, la alta proporción de barreras nos da un acercamiento importante a la necesidad de mejorar en la comunicación y señalización.

\section{REFERENCIAS}

1. Organización Mundial de la Salud. Informe Mundial sobre la discapacidad [Internet]. Ginebra: OMS; 2011. [citado 26 junio 2019]. Disponible en: https://www.who.int/disabilities/world_report/2011/summary_es.pdf

2. Instituto Nacional de Estadística (HN). La Discapacidad en Honduras: análisis de los resultados de la XXVI encuesta permanente de hogares de propósitos múltiples [Internet]. Tegucigalpa: INE; 2002. [citado 26 junio 2019].Disponible en: http://www.bvs.hn/Honduras/UICFCM/Discapacidad/ InformeDiscapacidadHonduras_INE_2002.pdf

3. Flores $\mathrm{S}$, Jimenez Dilworth $\mathrm{A}$, Castellanos Dubón W, Alger J, Zúñiga L, Gonzales M, et al. Prevalencia de discapacidad y sus características en población de 18 a 65 años de edad, Honduras, Centro América, 20132014. Rev Med Hondur [Internet]. 2015 [citado 29 octubre 2019];83(1,2):717. Disponible en: http://www.bvs.hn/Honduras/UICFCM/Articulo3_Vol831-2-Discapacidad.Sujetos.18-65a.pdf

4. Organización de las Naciones Unidas. La Agenda 2030 y los objetivos de desarrollo sostenible: una oportunidad para América Latina y el Caribe [Internet]. N.Y: ONU; 2020 [citado 12 agosto 2020]. Disponible en: www. cepal.org/es/suscripciones

5. Secretaria de Desarrollo e Inclusión Social (HN). Identificación y caracterización de las personas con discapacidad en Francisco Morazán: Sistematización de Honduras para todos [Internet]. Tegucigalpa: La Secretaría;
En conclusión, el diseño de las US en la red pública asistencial de Honduras en todos sus niveles, pero sobre todo en el nivel primario de atención, representa una barrera para la equidad en el acceso a la salud y se torna altamente relevante en el contexto de la pandemia de COVID-19: no permite la movilidad libre y autónoma para personas con deficiencia visual ni personas con movilidad reducida, particularmente los usuarios de sillas de ruedas, y para todas las personas hay serias limitaciones para cumplir con el distanciamiento físico. Para reducir la brecha en salud es necesario entre otras acciones, mejorar las condiciones físicas de los establecimientos donde se brinda la atención, a fin de que puedan ser accesibles para todas las personas y faciliten la integración de las personas con discapacidad.

\section{CONTRIBUCIONES}

AJD, WCD, SF, concibieron la idea original del estudio. AJD, WCD, SF, EM, JA, MG diseñaron el estudio y capacitaron a los recolectores de la información. AJD, WCD, SF, EM, JA, supervisaron el desarrollo del estudio en sus diferentes fases y contribuyeron al análisis de los resultados. EMA contribuyó en la supervisión de la recolección de datos, lideró la revisión de la Información digitada, el análisis de los datos y la elaboración del manuscrito. Todos los autores revisaron y aprobaron la versión final del manuscrito sometida.

\section{AGRADECIMIENTOS:}

A todos los estudiantes de último año de la Carrera de Medicina, cohorte 2013-2014, que participaron en la recolección de los datos, como parte del Seminario de Investigación impartido por la Unidad de Investigación Científica (UIC) de la Facultad de Ciencias Médicas (FCM) UNAH, Tegucigalpa, Honduras. A todo el personal de la UIC FCM UNAH y de la Asociación Hondureña de Medicina Física y Rehabilitación que ayudaron a supervisar la recolección de los datos.

2017 [citado 12 diciembre 2019]. Disponible en: https://bit.ly/3px60Aq. Organización de las Naciones Unidas. Convención sobre los Derechos de las Personas con Discapacidad y Protocolo Facultativo. N.Y.: ONU; 2007. [citado 12 diciembre 2019]. Disponible en: https://www.un.org/development/desa/disabilities-es/convencion-sobre-los-derechos-de-laspersonas-con-discapacidad-2.html

7. Organización Mundial de la Salud. Clasificación Internacional del funcionamiento de la discapadidad y la salud: CIF versión abreviada [Internet]. Ginebra: OMS; 2001. [citado 12 diciembre 2019]. Disponible en: https:// apps.who.int/iris/bitstream/handle/10665/43360/9241545445_spa.pdf;jses sionid=2221C7BA76285F32F780AA3C6D759B10?sequence=1

8. Iwarsson S, Stahl A. Accessibility, usability and universal design-positioning and definition of concepts describing person-environment relationships. Disabil Rehabil [Internet]. 2014[citado 12 diciembre 2019];1(2):14-5. Disponible en: https://journals.scholarsportal.info/pdf/09638288/v25i0002/57_ auaudadocdpr.xml

9. Isa Mosca E, Capolongo S. Towards a universal design evaluation for assessing the performance of the built environment. Stud Health Technol Inform. 2018;256:771-9.

10. Kuper $\mathrm{H}$, Hanefeld J. Debate: can we achieve universal health coverage without a focus on disability? BMC Health Serv Res. 2018;18(1):738.

11. Honduras. Congreso de la República. Ley de equidad y desarrollo inte- 
gral para las personas con discapacidad. Decreto No. 160-2005 [Internet]. Honduras: Congreso de la República; 2005. [citado agosto 21 2020]. Disponible en: http://www.unesco.org/education/edurights/media/docs/aec3346f7feab94d4d17b704a5569eaae2c46a9f.pdf

12. Secretaría de Desarrollo e Inclusión Social. Dirección General de Desarrollo para las Personas con Discapacidad. Plan nacional de accesibilidad universal. Tegucigalpa: DIGEDEPDI; 2008.

13. Frost KL, Bertocci G, Stillman MD, Smalley C, Williams S. Accessibility of outpatient healthcare providers for wheelchair users: Pilot study. J Rehabil Res Dev. 2015;52(6):653-62.

14. Siqueira FCV, Facchini LA, Da Silveira DS, Piccini RX, Thumé E, Tomasi E. Barreiras arquitetônicas a idosos e portadores de deficiência física: Um estudo epidemiológico da estrutura física das unidades básicas de saúde em sete estados do Brasil. Cienc e Saude Coletiva. 2009;14(1):39-44.

15. De Figueiredo Carvalho Z, Fontenele Moraes P de O, Agostinho Rolim $G$, De Almeida P. Accesibilidad en silla de ruedas en las clínicas y consultorios de neurología y neurocirugía de fortaleza - Brasil. Enferm Glob. 2008;14:1-14

16. Azevedo de, Rodrigues T, Costa S, Neyla de Freitas Macedo K, Valdevino J, Giovanna Rocha Silva de Holanda L, et al. Accesibilidad para personas con discapacidad física en los hospitales públicos. Enferm Glob. 2015;(37):310-8.

17. Elkartu, Federación Coordinadora de Personas con Discapacidad Física de Gipuzkoa (ES). Diagnóstico de accesibilidad en los hospitales de la CAPV para las personas con discapacidad. Vitoria-Gasteiz: Ararteko; 2017.

18. Cabrero García J. Disability indicators in the Spanish Disability, Impairment and Health Survey. Rev Esp Salud Pública [Internet]. 2007 [citado 21 agosto 2020];81(2):167-81. Disponible en: https://www.mscbs.gob. es/biblioPublic/publicaciones/recursos_propios/resp/revista_cdrom/vol81/ vol81_2/RS812C_167.pdf

19. Dassah E, Aldersey H, McColl MA, Davison C. Factors affecting access to primary health care services for persons with disabilities in rural areas: a "best-fit" framework synthesis. Glob Heal Res Policy [Internet]. 2018 [citado 21 agosto 2020 ];3(1):36. Disponible en: https://ghrp.biomedcentral. com/articles/10.1186/s41256-018-0091-x

20. Secretaría de Salud (HN). Modelo Nacional de Salud. Tegucigalpa: SESAL; 2013.

21. Bárcena A. Health protection as a citizen's right. Lancet [Internet]. 2015 [citado 18 agosto 2020];385:e29-30. Disponible en: https://www.thelancet. com/journals/lancet/article/PIIS0140-6736(14)61771-2/fulltext

22. Naciones Unidas. Comisión Económica para América Latina y el Caribe. Informes COVID-19. Personas con discapacidad ante la enfermedad por coronavirus (COVID-19) en América Latina y el Caribe: situación y orientaciones. Santiago: CEPAL; 2020.

23. Organización Panamericana de la Salud. COVID-19, Respuesta de la OPS/OMS: cifras clave de la respuesta de la Región de las Américas a la COVID 19 (del 4 de julio al 10 de agosto del 2020).Washington D.C.: OPS; 2020.

24. Pinheiro MD, Luís NC. COVID-19 Could Leverage a Sustainable Built Environment. Sustainability [Internet]. 2020[citado 21 agosto 2020];12(14):5863. Disponible en: https://www.mdpi.com/2071-1050/12/14/5863
ABSTRACT. Background. Universal Health Coverage is one of the Sustainable Development Goals. People with disabilities tend to have greater difficulties in accessing health care than other people. Environmental factors such as design and constructions can be a barrier or a facilitator. Objective. To evaluate the accessibility characteristics in design and mobility aids in health facilities where the students of the medicine faculty -National Autonomous University of Honduras,2013-2014 cohort -do their social labor prior to graduate. Methods. Based on universal design principles, barriers and facilitators for access to health were evaluated in health facilities, including primary care centers and hospitals. Results. 176 establishments in 17 departments, 154 primary care units (PCU) and 22 hospitals were evaluated. The biggest barriers were inadequate signaling of routes: 150 (97.4\%) PCU and $20(90.9 \%)$ hospitals; the lack of supports in the bathrooms: $152(98.7 \%)$ PCU and 20 (90.9\%) hospitals; inaccessibility to wheelchair users: $129(83.8 \%)$ PCU and 9 $(40.9 \%)$ hospitals. Discussion. Improving the access and quality of health services for people with disabilities is essential to reduce inequities. Our study indicates that the design of health facilities in Honduras represents a barrier to access services and healthcare quality for people with disabilities. The State is urged to regulate and supervise the design and construction of buildings accessible to all, in order to reduce health gaps.

Keywords: Architectural accessibility, Coronavirus infections, Health services accessibility, Health services for persons with disabilities, Universal health coverage. 